\title{
Clinical Efficacy of Nalbuphine Versus Tramadol as Analgesic Adjuvant to Fentanyl During Major Abdominal Surgery Performed Under General Anesthesia- A Double Blind Randomized Study
}

\author{
Abhishake Kumar ${ }^{1}$, Kumkum Gupta ${ }^{2}$, Prashant K Gupta ${ }^{3}$, Bhawana Rastogi ${ }^{1}$, Bhanupriya Agrawal ${ }^{4}$, Pavitra Kalra ${ }^{4}$ \\ ${ }^{1}$ Assistant Professor, Department of Anaesthesiology and Critical care, ${ }^{2}$ Professor, Department of Anaesthesiology and Critical care, ${ }^{3}$ Director and \\ Professor, Department of Radiology and Imaging, ${ }^{4}$ Resident, Department of Anaesthesiology and Critical care, Subharti Medical College, Swami \\ Vivekanand Subharti University, NH-58 Bye Pass Road, Meerut-UP, India.
}

\section{Abstract}

Background: Noxious stimulation of surgery predictably leads to variable hemodynamic changes which can be modified by opioid analgesia. The present study was aimed to comparatively evaluate the clinical efficacy of Nalbuphine with Tramadol as analgesic adjuvant to fentanyl during major abdominal surgery performed under general anesthesia. Subjects and Methods: Sixty adult consenting patients of ASA grade I and II of either sex, were enrolled for the study. Patients of Group I N received Nalbuphine $10 \mathrm{mg}$ and patients of Group II T received Tramadol $100 \mathrm{mg}$, intravenously, $15 \mathrm{~min}$ before induction of anesthesia. After propofol induction, the endotracheal intubation was facilitated by vecuronium bromide $(0.1 \mathrm{mg} / \mathrm{kg})$ and anesthesia was maintained with isoflurane and nitrous oxide with $40 \%$ oxygen. Changes in heart rate and systemic blood pressure were noted as primary variables and postoperative nausea, vomiting, respiratory depression, shivering or pruritus were noted as secondary outcomes. Results: Patients of comparable demographic profile showed fall in heart rate and blood pressure with no statistically significant difference. After extubation, patients of nalbuphine group were sedated but arousable while patients of tramadol group were awake. Five patients of tramadol group suffered from nausea. None of the patients of nalbuphine group suffered from any nausea. No patient showed any episode of respiratory depression, shivering, pruritus or any other side effects. Conclusion: Nalbuphine and tramadol, both could provide effective attenuation of the hemodynamic response to surgical stress of major abdominal surgery, but few patients of tramadol group suffered from manageable nausea.

Keywords: Nalbuphine; Opioid Analgesia; Hemodynamic Stress Responses of Surgery; Tramadol.

Corresponding Author: Prof (Dr) Kumkum Gupta, 108-109, Chanakyapuri, Shastri Nagar, Meerut-250004 U.P. India

Received: February 2019

Accepted: March 2019

\section{Introduction}

Surgical stress due to tissue injury, airway stimulation and pain initiate several physiological changes which may lead to variable hemodynamic changes of tachyarrhythmia and hypertension. Manipulation of abdominal contents during surgical procedure also caused hemodynamic variations. ${ }^{[1,2]}$ The magnitude of hemodynamic changes can be attenuated by using opioid analgesia, beta adrenergic blockers, alpha 2 adrenergic agonist, vasodilators, or by increasing the depth of anesthesia but with variable results. These agents are associated with their inherent side effects of respiratory depression, histamine release and gastrointestinal events.

Opioid analgesics act at presynaptic and post synaptic sites in the central nervous system to activate the pain modulating (antinociceptive) systems. Opioid receptors also exist on the peripheral ends of primary afferent neurons, where their activation may either directly decrease neurotransmission or inhibit the release of excitatory neurotransmitters. The major pharmacodynamics differences between the various opioids are their potency and rate of equilibrium between the plasma and the site of drug action. ${ }^{[3]}$

Fentanyl is synthetic opioid analgesic with activity as $\mu$ receptor agonist and is significantly more potent than commonly used opioids. The wide margin of safety, relatively short duration of action, ability to provide cardiovascular stability by blocking the stress response to surgical stimuli and minimal respiratory depression, has made it drug of choice.

Nalbuphine is synthetic $\kappa$ receptors agonist and $\mu$ receptor antagonist opioid analgesic and exert its action by opening the $\mathrm{K}+$ channels and reducing the $\mathrm{Ca}++$ influx which leads inhibition of transmitter release to block the nociceptive impulses from the surgical site. The advantage of opioid agonist-antagonist is its ability to produce analgesia with minimal respiratory depression and low potential to produce physical dependence. ${ }^{[4,5]}$

Tramadol is synthetic opioid analgesic with central effect. It possess weak agonist action at $\mu$ opioid receptor with additional mono-aminergic activity. Tramadol is also effective on noradrenergic and serotonergic 
neurotransmission which may add to its pain relief effects. The objective of this prospective double blind randomized study was to comparatively evaluate the clinical efficacy of nalbuphine with tramadol as analgesic adjuvant to fentanyl during major abdominal surgery performed under general anesthesia.

\section{Subjects and Methods}

\section{Selection Criteria}

The protocol of this prospective double blind randomized study was approved by Institutional Ethical Committee and written informed consent was obtained from each patient. The study was conducted on 60 otherwise healthy adult patients of American Society of Anaesthesiologist (ASA) physical status I and II aged 28 to 58 years of either sex and were scheduled for elective major abdominal surgery under general anaesthesia.

All patients underwent preanesthetic examination and patients with history of systemic hypertension or cardiac dysfunction, respiratory insufficiency, hemodynamic instability, hepatic or renal insufficiency, endocrine or metabolic disorder, morbid obesity, unstable personality and abuse liability were excluded from the study. Complicated surgeries of more than $2 \mathrm{~h}$ or patients taking any medication (antihypertensive, sedatives or analgesics) which could modify the stress response of surgery, were also excluded from the study.

\section{Randomization schedule}

Sixty enrolled patients were divided into two equal groups of 30 patients each according to a computer generated random number table. Allocation concealment was ensured with sealed opaque envelop. The study was conducted in doubleblind manner by use of coded syringe. Patients of Group I (N) were given Nalbuphine $10 \mathrm{mg}$ and patients of Group II (T) were given Tramadol $100 \mathrm{mg}$ intravenously, $15 \mathrm{~min}$ before induction of general anesthesia. Study medication was prepared by an anaesthesiologist by dissolving the study drugs in $10 \mathrm{ml}$ of normal saline. He was blinded to the randomization schedule and was not involved for data collection during study period to keep the blindness of study.

\section{Anesthetic Technique}

All selected patients were given tablet alprazolam $0.25 \mathrm{mg}$ and tablet ranitidine $150 \mathrm{mg}$ orally prior night before surgery and were kept fasted for 6 hours prior to surgery. They were operated during morning hours to minimize anxiety.

On the day of surgery, they received inj. glycopyrrolate 0.2 $\mathrm{mg}$ intramuscularly, 30 minutes prior to induction of anesthesia. On arrival to operation theatre, Multipara monitor was attached and baseline vital parameters of heart rate, systemic blood pressure, electrocardiogram and peripheral oxygen saturation $(\mathrm{SpO} 2)$ were monitored. An intravenous line secured and lactate Ringer solution was started at rate of 4-6 $\mathrm{ml} / \mathrm{kg} / \mathrm{h}$.

The patients of Group I (N) were given nalbuphine $10 \mathrm{mg}$ and patients of Group II (T) were given tramadol $100 \mathrm{mg}$, intravenously, in double blind manner, 15 minutes before induction of anesthesia. They were premedicated with ondansetron $4 \mathrm{mg}$, midazolam $2 \mathrm{mg}$ and fentanyl $2 \mu \mathrm{g} / \mathrm{kg}$, intravenously.
After 3 min of preoxygenation, anesthesia was induced with propofol $(2 \mathrm{mg} / \mathrm{kg})$, supplemented if required, till loss of verbal command. The laryngoscopy and intubation was facilitated with vecuronium bromide $(0.1 \mathrm{mg} \mathrm{kg}-1)$ and anesthesia was maintained with isoflurane and $60 \%$ nitrous oxide in oxygen. The patients were mechanically ventilated using closed circuit to maintain the normocapnia. The tidal volume and ventilatory frequency was adjusted to maintain $\mathrm{EtCO} 2$ between $35-40 \mathrm{~mm}$ of $\mathrm{Hg}$. The degree of muscle relaxation was maintained using the train of four ratio of $25 \%$ with supplemental doses of vecuronium bromide.

The patients were assessed for any changes in heart rate, blood pressure, and peripheral oxygen saturation along with analysis of electrocardiogram (ECG) for rhythm and ST segment changes. These parameters were recorded at baseline, before and after induction, immediately after intubation and then at $5 \mathrm{~min}$ interval during intraoperative period till end of surgery and post extubation.

The hemodynamic changes observed as abnormal finding during the study, were defined as hypotension when systolic blood pressure was less than $20 \%$ of baseline value or less than $90 \mathrm{mmHg}$, whichever was lower and hypertension was defined when systolic blood pressure was more than $20 \%$ of baseline value or more than $140 \mathrm{mmHg}$ whichever was higher. Tachycardia was defined as heart rate more than 100 beats/minute and bradycardia was defined as heart rate less than 50 beats/minute. Intraoperatively, any episodes of hypotension, hypertension, bradycardia, or tachyarrhythmia, was managed by adjusting the dial concentration of isoflurane and rate of lactate Ringer solution. Record of each such patient was kept.

At the end of surgery, isoflurane was discontinued and residual neuromuscular blockade was antagonized with neostigmine $(0.05 \mathrm{mg} / \mathrm{kg})$ and glycopyrrolate $(0.01 \mathrm{mg} / \mathrm{kg})$. Ventilation was continued to eliminate isoflurane until signs of awaking appeared. Patients were extubated after achieving signs of adequate reversal and he could obey the simple verbal commands along with return of regular, rhythmic respiration. All patients received injection ketorolac $30 \mathrm{mg}$, intramuscularly for postoperative analgesia.

\section{Postoperative follow up}

Patients were transferred to post anaesthesia care unit and monitored for any hemodynamic changes, respiratory depression, shivering, pruritus, or postoperative nausea and vomiting and managed accordingly.

\section{Study Population Size and Statistical Analysis}

The sample size was decided in consultation with statistician and was based on initial pilot observations which suggested that approximately 28 patients should be included in each group to ensure the power of study $80 \%$ and alpha error of 0.05 with confidence limit of $95 \%$ for detecting reduction by at least $20 \%$ in enhanced hemodynamic changes. Assuming a $5 \%$ drop out rate, the final sample size was set at 60 patients for better validation of results.

The data obtained in the study are presented in tabulated manner and variables are expressed as mean \pm standard deviation (SD), considering the later as the best predictor for statistical analysis. The results were analysed using Stat Graphic Centurion for windows, (Stat point technologies 
INC, Warrenton, Virginia). The parameters of both group were compared using Chi square test and unpaired T test. A $\mathrm{p}$ value of less than 0.05 was considered to indicate as statistical significance.

\section{Results}

The present study compared the clinical efficacy of intravenous nalbuphine with tramadol as adjuvant to fentanyl during major abdominal surgery performed under general anesthesia on 60 adult patients of both genders. The sample size was adequate to detect statistical significance. There was no protocol deviation and data of all patients were included for statistical analysis.

The demographic profile of age, weight, body mass index, gender ratio and ASA physical status were comparable between the groups. [Table 1]

Table 1: Showing demographic profile
\begin{tabular}{|l|l|l|l|}
\hline & Group I (N) & Group II (T) & P-value \\
\hline Age (year) & $41.37 \pm 10.2$ & $43.36 \pm 9.2$ & 0.67 \\
\hline Weight (Kg) & $54.63 \pm 5.6$ & $55.18 \pm 5.5$ & 0.49 \\
\hline BMI (Kg/m2) & $18.22 \pm 1.02$ & $20.27 \pm 0.7$ & 0.35 \\
\hline Gender (M/F) & $21 / 9$ & $23 / 7$ & 0.72 \\
\hline ASA (I/II) & $19 / 11$ & $22 / 8$ & 0.85 \\
\hline
\end{tabular}

Data expressed as Mean \pm SD, $\mathrm{P}$ value $>0.05$ is non-significant

\section{Hemodynamic Changes}

The hemodynamic parameters of heart rate and systemic blood pressure were monitored intra-operatively from induction till extubation and thereafter postoperatively.

The base line mean heart rate was comparable between the groups $(85.3 \pm 8.6$ vs $87.2 \pm 7.2$ beats $/ \mathrm{min})$. Patients of nalbuphine group showed fall in mean heart rate from base line till 10 minutes after induction with statistically significant difference between the groups. The difference in mean heart rate was maximal at 5 minutes after intubation. The mean heart rate in patients of nalbuphine group remained lower throughout the intraoperative period when compared to patients of tramadol group without any statistically significant difference. [Table-2]

Table 2: Showing Changes in Mean Heart Rate (beats/min)

\begin{tabular}{|l|l|l|l|}
\hline Time & Group I (N) & Group II (T) & P-value \\
\hline Base line & $85.3 \pm 8.6$ & $87.2 \pm 7.2$ & .067 \\
\hline Induction & $72.27 \pm 5.41$ & $76.67 \pm 6.5$ & 0.07 \\
\hline $5 \mathrm{~min}$ & $77.80 \pm 7.21$ & $85.40 \pm 6.8$ & $<0.05^{*}$ \\
\hline $15 \mathrm{~min}$ & $79.93 \pm 7.92$ & $87.12 \pm 6.02$ & $<0.05^{*}$ \\
\hline $30 \mathrm{~min}$ & $78.67 \pm 7.75$ & $84.11 \pm 7.9$ & 0.10 \\
\hline $45 \mathrm{~min}$ & $78.43 \pm 8.06$ & $87.90 \pm 7.48$ & 0.125 \\
\hline $60 \mathrm{~min}$ & $81.43 \pm 8.05$ & $88.4 \pm 7.81$ & 0.067 \\
\hline $90 \mathrm{~min}$ & $85.32 \pm 6.19$ & $91.80 \pm 5.74$ & 0.076 \\
\hline Post extubation & $87.47 \pm 8.35$ & $93.7 \pm 3.81$ & 0.063 \\
\hline
\end{tabular}

Data presented as Mean \pm SD; $\mathrm{P}$ value $<0.05$ is significant;

The mean systolic blood pressure at base line was comparable between the groups $(121.27 \pm 6.78$ vs 117.27 $\pm 7.41 \mathrm{~mm} \mathrm{Hg}$ ). The mean systolic blood pressure was minimal at induction in patients of both groups. It remained lower in patients of nalbuphine group when compared to tramadol group. The difference in mean systolic blood pressure decreased with time from induction till completion of surgery with no significantly significant difference between the groups. [Table-3]

Table 3: Changes in Systolic Blood Pressure
\begin{tabular}{|l|l|l|l|}
\hline SBP & Group I (N) & Group II (T) & P-value \\
\hline base line & $121.27 \pm 6.78$ & $117.27 \pm 7.41$ & 0.067 \\
\hline Induction & $109.27 \pm 6.741$ & $116.40 \pm 5.89$ & $<0.05^{*}$ \\
\hline $5 \mathrm{~min}$ & $116.87 \pm 11.13$ & $122.87 \pm 8.60$ & $0.004^{*}$ \\
\hline $15 \mathrm{~min}$ & $112.80 \pm 10.16$ & $117.47 \pm 6.882$ & 0.07 \\
\hline $30 \mathrm{~min}$ & $110.67 \pm 7.77$ & $112.87 \pm 5.251$ & 0.089 \\
\hline $45 \mathrm{~min}$ & $108.00 \pm 7.06$ & $112.80 \pm 5.36$ & 0.14 \\
\hline $60 \mathrm{~min}$ & $109.27 \pm 6.36$ & $115.27 \pm 4.55$ & 0.076 \\
\hline $90 \mathrm{~min}$ & $115.71 \pm 6.34$ & $119.27 \pm 7.83$ & 0.086 \\
\hline $\begin{array}{l}\text { Post } \\
\text { extubation }\end{array}$ & $124.91 \pm 4.36$ & $131.72 \pm 2.74$ & 0.062 \\
\hline
\end{tabular}

Data presented as Mean \pm SD; P value $<0.05$ is significant

The rescue analgesia was not required in any patient during intraoperative period. The postoperative hemodynamic parameters did not show any changes. Five patients of tramadol group suffered from nausea. No other patient in either group had any episode of hypotension, pruritus, shivering or respiratory depression in the postoperative period. No other complications related to study drug or anesthetic technique occurred during the study period.

\section{Discussion}

Surgical stress stimulation, endotracheal intubation and pain initiate sympathetic over activity, leading to increased blood pressure, heart rate, occasional dysrhythmias and plasma catecholamine concentration. Nociceptive pathways and humoral mediators, originating from the surgical site do enhance the adrenergic responses. ${ }^{[1,2]}$ Although these hemodynamic changes are transient but are detrimental in patients with pre-exiting myocardial or cerebral insufficiency. If these adverse hemodynamic responses are not attenuated, the postoperative outcome of the patient may be affected. Opioid analgesics, alpha 2-adrenergic agonist, beta adrenergic blocking agents and vasodilators could be used effectively to attenuate these intraoperative surgical stress responses.

Opioid receptors are located in areas of the brain and spinal cord which are involved with pain perception, integration of pain impulses and responses to pain. These receptors also exist on the peripheral ends of primary afferent neurons, resulting in activation of pain modulating (antinociceptive) systems. Opioids are unique in producing analgesia without loss of touch, proprioception or consciousness. ${ }^{[3]}$

The opioid receptors activation decreases the neurotransmission, mainly by presynaptic inhibition of neurotransmitter release, although postsynaptic inhibition of evoked activity may also follow. Administration of an opioid before surgical stimulation may decrease the subsequent amount of opioid required for postoperative analgesia.

The significance of study lies in the fact to select the better drug as an analgesic adjuvant to fentanyl for major abdominal surgery, which could attenuate the hemodynamic pressor response during period of stress, as both, nalbuphine and tramadol are opioid analgesics.

Nalbuphine is primary $\kappa$ agonist and $\mu$ antagonist and its analgesic potency is equal to morphine. Naloxone can reverse its agonist effects. Its affinity for $\kappa$ receptors produces analgesia and antishivering effects. Nalbuphine 
does not increase systemic blood pressure and heart rate, thus may be useful in providing sedation and analgesia for cardiac patients. Tramadol has weak agonistic action at $\mu$ opioid receptors with additional mono-aminergic activity. It is also effective on noradrenergic and serotonergic receptors. ${ }^{[6,7]}$

A single dose of fentanyl administered intravenously, has more rapid onset but shorter duration of action due to redistribution to inactive tissues. If given $5 \mathrm{~min}$ before induction of anesthesia, it decreases the subsequent doses of isoflurane to block the sympathetic responses to surgical stimulation.

The precise mechanism that leads to hemodynamic changes involve intense sympathetic discharge and release of catecholamine. In the present study, after administration of fentanyl with either nalbuphine or tramadol, there was fall in mean heart rate and systolic blood pressure in patients of both groups with no statistically significant difference between the groups. After induction, the difference in heart rate changes was statistically significant between the groups, but decrease in systolic blood pressure was more evident in patients of nalbuphine group.

The heart rate was increased during laryngoscopy and intubation and was more evident in patients of tramadol group when compared to patients of nalbuphine group. The difference between the groups was statistically significant till 5 minutes after intubation. It was evident from the present study that nalbuphine was able to attenuate hemodynamic response of airway stimulation.

In patients of nalbuphine group, the initial fall in all the hemodynamic parameters was due to its strong and predominant kappa agonistic action. Increase in hemodynamic parameters after endotracheal intubation was due to sympatho-adrenal stimulation of pharyngeal structures during direct laryngoscopy.

Ahsan-ul-Hag et al also compared nalbuphine with placebo and observed rise in heart rate and mean arterial pressure just after intubation in placebo group which was significant from baseline while nalbuphine prevented this rise. Their observations are in concurrence of the present study. ${ }^{[8]}$

Peak effects of nalbuphine are seen approximately $20 \mathrm{~min}$ after its administration which could be seen in present study as the heart rate and blood pressure started to return towards baseline approximately $5 \mathrm{~min}$ after intubation, whereas in tramadol group, the hemodynamic pressor response was sustained up to $15 \mathrm{~min}$ post laryngoscopy.

Various studies have also concluded that fentanyl and nalbuphine are effective in keeping the patients hemodynamically stable and the results of present study are in accordance with previous clinical studies. ${ }^{[9,10]}$

Chestnut et al compared the effects of nalbuphine, pethidine and placebo. They noticed excellent control of hemodynamic response during gynaecological surgery in patients of nalbuphine and pethidine group, but noticed nausea and vomiting at the end of surgery which was more in patients of pethidine group. ${ }^{[11]}$ Kothari and Sharma also used nalbuphine and noticed effective reduction in heart rate and mean arterial pressure as compared to pentazocine. ${ }^{[12]}$ The present study also supports their results.

In the present study, intravenous nalbuphine or tramadol before induction of anesthesia, has modified the hemodynamic pressor responses of laryngoscopy and surgical stimulation but did not totally abolish them. The variations of blood pressure and heart rate never exceeded more than $15 \%$ of baseline which could be attributed to their effective analgesic potency.

Hypotension and bradycardia was not observed in any patient during the study period, hence intravenous atropine or vasopressor was not used. This may be because of adequate pre-anesthetic plasma volume expansion and intramuscular glycopyrrolate premedication.

Chung et al and other researchers observed that pure opioid agonists can cause complications such as respiratory depression which can be dangerous in the recovery room. ${ }^{[13,14]}$ Five patients of tramadol group had episode of nausea. On the other hand, nalbuphine is agonist-antagonist opioid and cause less respiratory depression by acting on the supraspinal and spinal kappa receptors. There is lower incidences of postoperative respiratory depression, pruritus and nausea and vomiting (PONV) with nalbuphine when compared to morphine, as observed by many researchers. ${ }^{[14,15]}$

\section{Conclusion}

Nalbuphine has more effectively attenuated the stress response of laryngoscopy and surgical stimulation when compared to tramadol, but both drugs provided valuable intraoperative analgesia for major abdominal surgery performed under general anesthesia.

\section{References}

1. J P Desborough. The stress response to trauma and surgery. British J Anaesthesia 2000; 85: 109-17.

2. Singh M. Stress response and Anesthesia, Altering the perioperative and postoperative management. Ind J Anaesth 2003; 47: 427-34.

3. Andrea M Trescot, Sukdeb Datta, Marion Lee, Hnas Hansen. Opioid pharmacology. Pain Physician 2008; 11: S 133-53.

4. Opioids FK. Nalbuphine. Miller's Anesthesia 7th ed. Philadelphia PA: Churchill Livingstone, Elsevier; 2010: page 809.

5. Priti M Chawda, Mayuresh K arrek, Ketan D Mehta. Effect of Nalbuphine on Hemodynamic Response to orotracheal intubation. J Anaesth Clin Pharmacol 2010; 26: 458-60.

6. Mark W Gunion, Anna Maria Marchionne, Corrie TM Anderson. Use of the mixed agonist-antagonist nalbuphine in opioid based analgesia. Acute Pain 2004; 6: 29-39.

7. De Souza EB, Schmidt WK, Kuhar MJ. Nalbuphine: An autoradiographic opioid receptor binding profile in the central nervous system of an agonist/antagonist analgesic. J Pharma Exp Ther 1988; 244: 391-402.

8. Ahsan-ul-Haq M, Kazmi EH, Rao ZA. Nalbuphine prevents hemodynamic response to endotracheal intubation. J Coll Physiciam Surg Pak 2005; 15: 668-70.

9. T J Gal, C A DiFazio and J Moscicki. Analgesic and respiratory depressant activity of nalbuphine: a comparison with morphine. Anaesthesiology 1982; 57: 367-74.

10. F N Minai, F A Khan. A comparison of morphine and nalbuphine for intraoperative and postoperative analgesia. J Pakistan Medical association 2003; 53: 2003.

11. Chestnutt WN, Clarke RSJ, Dundee JW. Comparison of nalbuphine, pethidine and placebo as premedication for minor gynaecological surgery. Br J Anaesth 1987; 59: 576-80.

12. Kothari D, Sharma CK. Effect of nalbuphine and pentzocine on attenuation of hemodynamic changes during laryngoscopy and endotracheal intubation: A clinical study. Anaes Essys Res 2013; 7: 326- 30 .

13. Chung W, Ko Y, Yoon $\mathrm{H}$ et al. Effect of nalbuphine on hemodynamic values and Bispectral indices during total intravenous anesthesia with 
propofol and remifentanil. Korean J Anaesthesiol 2007; 53: 7-11.

14. Shiv Akshat, Rashmi Ramachandran, Vimi Rewari, Chandralekha,

Anjan Trikha, Renu Sinha. Morphine versus Nalbuphine for open gynaecological surgery: A randomized controlled double blinded trial.

Pain Research and Treatment 2014, http://dx.doi.org/10.1155/2014/727952.

15. A Romagnoli, A S Keats. Ceiling effect for respiratory depression by nalbuphine. Clinical Pharmacology and Therapeutics 1980; 27: 47885

Copyright: (C) the author(s), publisher. Academia Anesthesiologica International is an Official Publication of "Society for Health Care \& Research Development". It is an open-access article distributed under the terms of the Creative Commons Attribution Non-Commercial License, which permits unrestricted non-commercial use, distribution, and reproduction in any medium, provided the original work is properly cited.

How to cite this article: Kumar A, Gupta K, Gupta PK, Rastogi B, Agrawal B, Kalra P. Clinical Efficacy of Nalbuphine Versus Tramadol as Analgesic Adjuvant to Fentanyl During Major Abdominal Surgery Performed Under General Anesthesia- A Double Blind Randomized Study. Acad. Anesthesiol. Int. 2019;4(1):76-80.

DOI: dx.doi.org/10.21276/aan.2019.4.1.17

Source of Support: Nil, Conflict of Interest: None declared. 